\title{
International Mortality and Longevity Symposium
}

[Institute and Faculty of Actuaries, Royal Holloway, University of London, 7-9 September 2016]

\section{Introduction}

\section{Joseph Lu, Director Longevity Science, Legal \& General}

This 3-day International Mortality and Longevity Symposium brought together thought leaders across relevant disciplines to discuss the latest thinking on the drivers and future of mortality trends in populations.

Our attendees included actuaries, demographers, modellers, medical scientists and epidemiologists. We have gained new insights and learned new techniques from our distinguished experts in seven plenary sessions, 16 workshops and four representatives from the IFoA's Actuarial Research Centre. Please click this link for their presentations: https://www.actuaries.org.uk/learn-and-develop/ events-calendar/past-events/international-mortality-and-longevity-symposium-2016

\section{Biology of ageing}

Our plenary speakers discussed the latest developments on the trend in life expectancy, biology of ageing and potential intervention of the process of ageing. Life expectancies at birth in most developed countries have been rising, first driven by reduction in early and mid-life mortality rates in the two centuries leading to around 1970s and then by reduction in older-age mortality rates until today. However, some developed nations have experienced a notable decline in life expectancy or deceleration in the rate of increase in recent years. Hence, a good understanding of what drives longevity and ageing in middle and older ages is important in forecasting life expectancy. Scientists now consider ageing to be the result of accumulation of defects in the cells and molecules in our bodies, rather than of a biological programme that drives us to death. Researchers have differing views on the potential likelihood and impact of age-retardation technology on longevity, but there is broader agreement that priority should be placed on improving health rather than extending lifespan. The biological process of ageing is complex, so the development of a "silver bullet" to retard ageing faces challenges. There is scope for actuaries to consider the potential and challenges of age-retardation technology in impacting life expectancy, when they forecast population longevity.

\section{Bigger data for better decisions}

Many actuaries are responsible for risk assessment of life insurance products, pension plans or social security schemes that involve payments in the event of illness, death or survival of people. So, data on health, disease and death are indispensable for actuarial work. The rapid progress in computing power means that richer data sets containing health and mortality data are now captured in the public and private sectors. A plenary session discussed the plethora of databases that could contribute to actuarial decisions. However, access to these health databases can be hindered by a lack of public trust, coherent regulation and effective collaboration between the public and private sectors. Therefore, there is a need to address these issues so that the public can benefit from improved products and services as a result of insights from these databases. 


\section{Socio-economic implications of longevity}

The combination of rise in life expectancy and drop in birth rates has led to increasing proportion of older population in the United Kingdom - a phenomenon called the ageing population. For example, the median age in the United Kingdom has risen from 34 in 1974 to about 40 in 2014 (Government Office for Science, 2016). More than 70\% of the rise in UK population between 2014 and 2039 is expected to come from the above 60 age group, an increase from 14.9 to 21.9 million people (Government Office for Science, 2016). The plenary sessions discussed how the ageing population will have a far-reaching impact on health care, public spending and intergenerational financial relationships. Although older people are living longer, they are also living longer with disability and illness in the UK and several EU countries. This will increase national spending on health and social care.

The UK's public spending on state pension will increase if all aspects of pension provisions remain the same. However, revisions of criteria for pension age and pension increases could be used to manage spending costs. Intergenerational inequality in wealth distribution would have long-term fiscal impact. For example, the proportion of homeowners for people aged 25 has dropped from $45 \%$ for those born in 1963-1967 to 20\% for people of the same age who were born in 1983-1987. The younger generations are less likely to have an occupational pension. Additionally, they have seen lower wage growth partly due to redistribution of resources to fund the pension costs of the older generation. Taken together, much more needs to be done to harness the benefits and tackle the challenges of ageing populations.

\section{Conclusion}

The symposium explored examples of when actuarial work can benefit from closer collaboration with other disciplines including medical sciences, demography and data sciences. There were plenty of opportunities for networking. I trust the knowledge, skills and network that have emerged from this symposium will help you advance your professional and research goals.

\section{Plenary sessions}

\section{Can we live forever?}

\section{Professor Tom Kirkwood, Professor Emeritus, Newcastle University Institute for Ageing and Professor of Biogerontology, University of Copenhagen Center for Healthy Aging}

Humanity's enduring fascination with the idea of immortality means that any advance in research on ageing tends to attract headlines such as "scientists discover secret of youth!" The reality is more prosaic. Ageing is a complicated process and the sum of what we know is still dwarfed but what remains unknown.

Nonetheless, life expectancy is still increasing, driven not by the time-honoured reductions in early and mid-life death rates of the last two centuries, but by rapid decline in death rates of those who are old already. We tend now to reach old age in better condition than did our ancestors, if indeed they 
reached old age at all. If ageing is amenable to improvement, as is apparently the case, what possibilities exist further to extend our period of good quality life?

Biology reveals that there is no strict programme driving us to die, but that ageing is caused by the gradual, lifelong build-up of small faults in the cells and molecules that make up our bodies. Genetics plays its part, by regulating the quality of our maintenance systems, but genes account only for a quarter of what influences the individual's length of life. The rest comes from things like nutrition, lifestyle, education, housing, attitude and probably much else besides.

It might turn out that scientific discoveries will one day boost our chances to enjoy health for longer, but we should be honest in acknowledging the significant challenges that will first need to be surmounted. Most of all, we need a massive effort to bring the science of ageing to fruition in helping us to learn much more about age-related illness. What is it about the mere fact of getting older that so greatly increases the risk of developing any one of the many chronic diseases for which age is much the greatest risk factor?

\section{The rise and fall of human longevity in the $21^{\text {st }}$ century}

\section{S. Jay Olshansky, PhD, University of Illinois at Chicago}

Olshansky started by identifying the rise in human longevity in the last 120 years as one of humanity's major accomplishments. The good news is that we're living longer because in the absence of fixed genetic programmes for ageing or death, it has become possible to modify the world around us to foster conditions that favour greater longevity.

There is reason to be optimistic that future advances in the biomedical sciences will not just continue, but they are likely to accelerate - leading to the manufacture of additional survival time. Just how much longer medical technology can make us live has yet to be determined.

The challenging news is that our increased longevity is accompanied by a Faustian trade - we now live with regularity into an age window where the biological processes of ageing remain immutable, and the fatal and disabling diseases that accompany our longer lives rise exponentially with greater longevity. It is possible, perhaps likely, that increased longevity brought forth by the medical advances mentioned above, could yield negative health trade-offs soon. That is, gains made against cardiovascular diseases and cancer could expose the saved population to an elevated risk of Alzheimer's disease and other negative trade-offs that are undesirable and costly.

Olshansky was optimistic that interventions that modulate biological ageing are forthcoming (he is personally supporting these ideas through the Longevity Dividend Initiative), but the biological reality is that human bodies were not built to last. There is indeed excitement in the air about ageing science, but this excitement must be tempered by the biological reality and recently observed trends in mortality in long-lived populations suggesting that linear increases in life expectancy are neither biologically nor demographically justifiable - especially not in the United States. Recent trends in life expectancy in other long-lived populations, such as the United Kingdom, France and Canada, suggest that we may be approaching a point of diminishing returns. Actuaries and demographers need to think very carefully about their forecasting assumptions considering both Faustian trade-offs and the underlying biology that determine lifespan potential. 


\section{Health and longevity - can we have both?}

\section{Professor Carol Jagger, AXA Professor of Epidemiology of Ageing and Deputy Director, Newcastle University Institute for Ageing, Newcastle upon Tyne}

Professor Jagger's session covered trends in life and health expectancy in the United Kingdom since 2000, making comparisons with four European countries (Belgium, France, the Netherlands and Sweden) and three OECD countries (Japan, Switzerland and the United States).

Trends in life expectancy (LE) at birth were similar for all countries considered although UK women's life expectancy is the lowest of the four European countries and only US women's life expectancy is lower.

Men's life expectancy at birth, on the other hand, ranks higher. Whether these extra years of life are healthy ones is the question that health expectancies can answer. Professor Jagger focussed first on the two main health expectancies routinely reported by the Office for National Statistics: Healthy Life Expectancy (HLE) based on self-rated health; and Disability-free Life Expectancy (DFLE) based on activity restriction. In the United Kingdom since 2000 there appears to have been some compression of disability and ill-health at younger ages, with increases in DFLE and HLE at birth being larger than increases in life expectancy. However, the opposite is true at older ages, 65 and 85, so that the extra years of life are spent with disability of ill-health. Trends in DFLE and HLE in other countries are much more variable than LE with only Sweden and Switzerland showing a compression of disability or morbidity at all ages.

Professor Jagger then described the most recent findings for England, using the Cognitive Function and Ageing Studies, on a wider range of health expectancies at age 65. She demonstrated that conclusions about whether or not the extra years of life were healthy ones depends heavily on the measure of health. There was a real compression of cognitive impairment between 1991 and 2011, in keeping with the reduction in the prevalence of dementia over the same time period, and also seen in Sweden and the United States. However, the picture for disability was much less positive with an expansion of mild disability, a finding also evident in the United States.

Finally, Professor Jagger discussed the possible drivers of trends in DFLE in terms of health behaviours (obesity, smoking, physical inactivity) and chronic conditions. The discussions that followed covered whether the smaller improvements in HLE compared to LE were perhaps due to individuals being diagnosed earlier especially around particular targets for general practitioners. Professor Jagger replied that this might be a contributing factor but the self-rated health on which HLE was based was a more holistic measure and would also encompass mental as well as physical health.

\section{Public data for the private sector}

\section{Dame Karen Dunnell, Chair, Longevity Science Panel}

Dame Dunnell began by explaining that The Longevity Science Panel of Legal and General are active users of statistical information and databases of all kinds. It was the crisis over the failure of the care. 
Data programme that led the panel to carry out their project to understand what went wrong and to make recommendations about how to improve the situation.

The insurance industry and actuaries have always had access to anonymised information from administrative sources like birth and death registration and Hospital Episode Statistics. Census and survey data are also available via the Economic and Social Research Council (ESRC, UK). Access to this data is becoming more difficult.

Using the examples of an ageing population and its social care and housing needs Dunnell showed how access to a wider range of publicly collected information could benefit the development of services and products for our ageing society.

The panel recommend:

- The formation of a publicly acceptable legal structure and entity to govern the collection, management and dissemination of public statistics, data and information to support the public good.

- The "public good" be defined in such a way that includes any activity that benefits society or the economy, particularly where that activity is aligned with public policy.

- Strategies are employed to improve the appropriate access of both the public and private sectors to all publicly funded data, information and statistics, while protecting the privacy of individuals and the public interest.

- Private sector and public bodies are included in discussions to specify what public data and information are needed for the benefit of society and the economy.

\section{Demographic change and fiscal policy}

\section{Paul Johnson, Director of the Institute for Fiscal Studies}

The United Kingdom is ageing. This matters to fiscal policy because older people are less likely to be in employment and spending on older people is much higher than on younger people. To cope with an ageing population the government will either have to increase taxes, or reduce spending on the health and pensions of older people, or cut spending on other services - which have been hit particularly hard by cuts in recent years.

\section{Pensions}

The fiscal impact from increased state pension spending is actually fairly manageable. Increases in the state pension age, reflecting increased longevity, and ending the unsustainable "triple-lock" whereby pensions rise by the faster of inflation, earnings growth and $2.5 \%$ - could keep additional spending on pensions down to as little as an extra $1 \%$ of national income despite the rapid growth in numbers of older people.

\section{Health care}

The impact of ageing on health care spending is more uncertain and the additional cost will likely be much higher than the additional cost of pension provision. The affordability of health care spending depends not just on demographic change but is also highly sensitive to what happens to productivity 
in the health service. If productivity rises only as fast as it has over the last 50 years we could easily see spending rise by $5 \%$ of national income over the next 40 or 50 years.

\section{Intergenerational equity}

Those retiring over the last decade or so and those retiring in the next decade have been very fortunate economically. They have done well in the housing market and many have generous occupational pensions. Younger people are much less fortunate. There are no defined benefit occupational pensions left in the private sector and home ownership rates have collapsed as prices have risen. Prolonged low interest rates have also made retirement savings much more challenging than for previous generations. At the same time earnings of those in their twenties are lower than for those at the same age more than a decade ago. The younger generation is likely to be particularly dependent on inheritance, reducing social mobility. Policy needs to reflect the reduced economic resources of this generation.

\section{Macro-demographic perspective of longevity}

\section{Dr Amlan Roy, Systemic Risk Centre, London School of Economics and Guest Finance Professor, London Business School}

The goal of this plenary session was to highlight the important insights from quantitative finance, investment theories, macroeconomics and behavioural finance towards a contribution to the existing strong background of actuaries in the world of pensions. This was similar to a closing plenary speech at the Investment Symposium of the Society of Actuaries. Understanding the big picture requires the actuarial profession to integrate insights from the academic and applied finance/portfolio investments literature.

Dr Roy started by challenging the traditional age classifications of old, working age and young used by economists and actuaries demonstrating that the fastest growing age group and the one responsible for ageing related fiscal sustainability issues is the $80+$ age group. He cited management and pensions global guru Drucker (1999) who argued that most people do not understand demographics as they take a narrow focus. Dr Roy argues that demographics is about people characteristics, of which age, is just one of the characteristics. He urged for a broader understanding of demographics pertinent to individual characteristics as consumers and workers which underlie many facets of defined contribution and hybrid pension schemes.

He highlighted significant divergences in longevity and demographic trends across rich countries cautioning against broad generalisations. He raised issues regarding combining different statistical techniques to arrive at longevity forecasts (Haberman, 2016) and also a better understanding of international longevity differences along with their impact and effects (Gillespie et al., 2016 [2014]).

He demonstrated the challenges of global growth and showed how demographic factors affect gross domestic product (GDP) growth and fiscal sustainability of the European Union, Japan and the United States. The vast demographic divergences across emerging nations of Brazil, China, India, Russia, Mexico and Turkey were highlighted in the context of the demographic dividend theory which posits contribution of demographics to GDP per capita. 
Also illustrated were the bigger issues of youth unemployment and gender disparity across countries and regions of the world. Their amelioration (or elimination) is essential for unblocking the growth and equity prospects of the world. The role of macro demographics in influencing interest rates and the ineffectiveness of current monetary policy was argued.

Quantitative links of demographics to savings, investment and current account were illustrated in order to explain the global linkages in an interconnected world which is changing in a post Brexit and likely post Trump world. Further quantitative investment implications through sectors that were demographically advantaged and macro-asset allocation changes were also stressed.

In conclusion, Dr Roy stressed the need to understand dynamic macroeconomics, portfolio theory, quantitative finance and behavioural economics is critical for actuaries, investors and policy-makers as the top-down dynamic effects are critical for strategic asset allocation and strategic Asset Liability Management. This would complement the existing actuarial and biomedical approaches to pensions.

\section{Causal model for mortality, morbidity and longevity}

\section{Dr Chris Martin, Clinical Modelling Consultant at L\&G. Joseph Lu, Director, Longevity Science, Legal \& General. Matthew Edwards, Head of Mortality and Longevity, Life Insurance Practice, Willis Towers Watson}

Dr Chris Martin focussed on causal models for mortality, their use in medicine and how they might translate to the retirement products domain. He began by outlining the history of mathematical and computer modelling in the medical literature and how it has developed over time.

He described medical uses of regression models, Bayesian systems, neural networks, Markov models, "expert systems" and other logical rule-based methods, as well as discrete event simulation. He finished with tips on how to exploit the medical literature for parameterisation of cause-of-death models.

In summary, regression models had clearly dominated the field and continued to do so. Interest in early rule-based "expert systems" was in relative decline, though newer methods such as classification and regression trees had potential for application in underwriting. Looking to the future, discrete event simulation is used increasingly in the modelling industry supporting economic evaluation of new drugs, and may supplant Markov modelling as the dominant method.

Joseph Lu discussed some emerging drivers that offer opportunities for actuaries to serve the public through providing mortality, morbidity and longevity assumptions. These drivers include:

- Increasing demand for de-risking of the global-defined benefit pension funds, estimated at $\$ 10$ trillion. Actuaries will need to estimate current future mortality rates of pensioners in different socio-economic groups.

- In the United Kingdom, average age is rising, but people are not saving enough. Over 60s have c. $£ 1.3$ trillion in housing equity, increasingly used to fill saving gaps. Examples of newer products include lifetime mortgage that requires assumptions for care home entry and mortality rates of customers. 
- Pressure on public finances is moving the provision of welfare from the state to individuals and employers, needing private provision of protection products such as critical illness, income protection and life insurances.

- Rising wealth in developing countries with less comprehensive state health care provision.

Projections of future longevity could draw upon techniques from a range of fields, including statistics, medical science, epidemiology and demography. Population trends can also be broken down into subpopulations to examine mortality rates according to gender, socio-economic status or health condition. There are opportunities for actuaries to work with other disciplines to develop forecasting methods that take account of drivers of longevity and morbidity.

Matthew Edwards discussed his recent development of a disease-based Markov model simulating the mortality process through transitions from healthy to disease to death, or from other starting points. This has obvious uses in a medical underwriting context, but can also add insights in the field of longevity improvements. Through a pool of medical experts, the likely improvement trend for each transition (for instance, from healthy to diabetes, or from stroke to death) can be quantified with suitable rationale to allow proper discussion as to the suitability of the assumptions. The model then provides a framework to combine those trends across all diseases and all state transitions.

Matthew illustrated some of the results obtained from this work. For instance, the model was able to quantify the expected female mortality improvements as a proportion of male improvements (circa $60 \%-90 \%$ over a typical pensioner age range), and also able to segment the proportions of overall improvements attributable to improvements in morbidity as opposed to improvements in mortality from the disease state (the split was $\sim 15 \%$ morbidity: $85 \%$ mortality).

\section{Workshop sessions}

\section{Coherent mortality projections for the Netherlands taking into account mortality delay and smoking}

\section{Fanny Janssen, Population Research Centre, University of Groningen, the Netherlands}

Recent analyses of mortality trends reveal a transition from mortality compression (a changing shape of the age-at-death distribution with more deaths occurring around the modal age at death) towards mortality delay (a shift in the age-at-death distribution towards older ages) (e.g. Janssen and de Beer, 2016). Mortality projections including this shift have been applied before, albeit very rarely, and only for single populations/countries. Also these mortality projections did not account for the effect of smoking, whereas smoking is known to greatly affect future mortality trends and levels (e.g. Janssen et al., 2013). Recently developed coherent mortality projections have not been applied much, let alone in combination with smoking and mortality delay.

The speaker proposed a mortality forecasting methodology which simultaneously takes into account the effect of smoking, mortality delay and mortality compression, and the mortality experience of the opposite sex and of other countries. She illustrated the methodology with a mortality forecast for the Netherlands. See Janssen \& de Beer (2016) for more details. 
For the Netherlands, the necessity of taking into account smoking when performing projections based on mortality delay clearly showed. That is, compared to all-cause mortality, for non-smokingrelated mortality increases in the modal age at death - indicating mortality delay - are much more linear, and more similar for men and women. The coherent mortality projection for the Netherlands which took into account both mortality delay and smoking resulted in higher remaining life expectancy at age 40 in 2050 and more deaths at higher ages compared to a conventional (Lee-Carter) projection and the forecast by Statistics Netherlands. The Lee-Carter model seems not able to fully capture the (continued) delay.

\section{Extreme scenarios for pandemic risk}

\section{Dr Gordon Woo, Catastrophist, Risk Management Solutions}

Dr Woo gave a presentation on extreme scenarios for pandemic risk. He started by pointing out that it is almost a century since the last catastrophic influenza pandemic. The most recent influenza pandemic was also H1N1, and occurred in 2009. Because this was mild, there is a psychological outcome bias towards people downgrading pandemic risk as a cause of mass excess mortality.

Dr Woo stressed that in order to understand the scope of pandemic risk, the spectrum of possible future scenarios needs to be constructed based on the current human population and infectious disease environment. This structured approach to dynamic modelling is more insightful for risk management than a purely statistical method. Intrinsic to this approach is the maximal incorporation of medical and public health knowledge.

Of particular actuarial interest for life insurers are the extreme pandemic scenarios. A benchmark for a pandemic of insurance catastrophe proportions is the 1918 pandemic. Many more died of the influenza pandemic than in the Great War itself. Dr Woo made a special note, which is significant for risk assessment, that the two global disasters were causally connected. The influenza was brought to the western front by a cohort of 100,000 Chinese labourers despatched there by the Chinese government seeking favour from the western powers.

The nexus between political conflict and a global pandemic provides one clear route to disaster. Dr Woo was asked about current extreme scenarios of this kind. In response, he cited the counterfactual possibility in 2015 that an emerging Middle East respiratory syndrome outbreak in the Middle East might have developed into a pandemic through the migration of a million refugees into Europe. He also commented that it was fortunate there was no civil war in West Africa when Ebola struck in 2014.

\section{Medical innovation versus Risk factors: a future perspective on breast and lung cancer}

\section{Nicola Oliver, Medical Director and Head of Longevity and Mortality Research, Medical Intelligence (UK) Ltd}

The last 30 years have witnessed significant advances in medical treatments for some, but not all, cancers. Similarly, changes in lifestyle behaviour risk factors, both positive and negative, have 
affected disease risk. Breast cancer and lung cancer were selected for closer scrutiny due to their contrasting profiles in terms of survival and mortality.

In the first half of this session, the impact of medical treatments, specifically combination therapy and targeted therapy, in the management of breast and lung cancer were explored in order to understand the impact of treatments on prognosis.

Breast cancer is generally diagnosed at early stages thus enabling timely and effective treatment, as compared with lung cancer, in which late stage diagnosis results in poor prognosis, survival and high mortality rates. This has resulted in improving mortality rates for breast cancer over time, and, ironically, increasing incidence as many more cancers at early stages are identified. Lifestyle risk factors such as obesity and smoking rates were discussed in relation to their impact on disease risk.

In the latter part of the session, the future impact of risk behaviours including smoking, obesity, alcohol intake and sedentary behaviour were discussed in relation to their impact on disease risk and survival. Their impact was examined against the backdrop of improving medical innovation and diagnostics.

In conclusion, obesity and smoking represent continuing threats to future disease risk; however, potential modifying factors including improved screening and diagnostics and the ever expanding impact of targeted therapies will potentially triumph over the negative impact of lifestyle risk behaviours, particularly in light of continued public health efforts to combat smoking and obesity rates.

\section{Use of routinely collected primary care data to model longevity and longevity improvement}

\section{Professor Elena Kulinskaya and Lisanne Gitsels, University of East Anglia}

This workshop session was focussed on how statistical modelling of population-based health data measured at individual level could be used to assess longevity risks and how actuarial data could then be used to translate the results to the population of interest to the actuarial community.

Professor Kulinskaya discussed her new research programme (2016-2020) funded by the Institute and Faculty of Actuaries (IFoA). The aims of the research programme are to identify and quantify the key factors affecting mortality and longevity, such as lifestyle choices, medical conditions and/or interventions; modelling of temporal changes in the factors affecting morbidity and mortality; evaluation of plausible scenarios in mortality trends of insureds due to medical advances or lifestyle changes; and development of software tools to forecast longevity risk. The aims will be addressed using The Health Improvement Network (THIN) primary care data and the Continuing Mortality Investigation actuarial data.

Lisanne Gitsels discussed a case-study on longevity improvement attributable to the widening of the prescription of statins under recently revised National Institute for Health and Care Excellence criteria. The results are based on the preliminary research within Professor Kulinskaya's research 
programme and published in Gitsels et al. (2016). Using THIN data, four cohorts aged 60, 65, 70 or 75 years with no previous history of cardiovascular disease were studied, with sample sizes 118,700, 199,574, 247,149 and 194,085, respectively. The results demonstrated that the already eligible patients (QRISK2 of $\geq 20 \%$ ) had a life expectancy improvement of $14 \%-18 \%$ if prescribed statins by the age of 65 , the newly eligible patients (QRISK2 of $10 \%-19 \%$ ) had an improvement of $11 \%-21 \%$ if prescribed statins by the age of 70 , and the non-eligible patients (QRISK2 $<10 \%$ ) had no improvement by any age. The improvements attributable to statin use translated to an increase in life expectancy of $1.2-2$ years.

\section{Inferences for maximum country life expectancy using provincial data}

\section{Anthony Medford, Max Planck Odense Centre on the Biodemography of Aging, University of Southern Denmark}

The most popular mortality forecasting models, the Lee-Carter Model (Lee \& Carter, 1992) and its numerous extensions and variants fit trends to age-standardised $(\log )$ death rates. However, there is a strong argument for using life expectancy in forecasting. White (2002) found that linear trends in life expectancy give a better empirical fit to the experience of individual countries than linear trends in age-standardised $(\log )$ death rates in his study of 21 developed countries.

Global Best Practice Life Expectancy (BPLE) is the maximum life expectancy observed among nations at a given age. Can the notion of BPLE be could be extended to regions smaller that the global whole and what kinds of conclusions can be drawn? A new approach to modelling and projecting (maximum) life expectancy for an arbitrary region using only data from the sub regions was explored by applying ideas from the statistical theory of Extreme Values. The method was applied to the Canadian provinces and Japanese prefectures using high-quality data from the Canadian and Japanese Mortality Databases.

One advantage of the approach is that it is possible to make probabilistic statements about future maximum Canadian life expectancy. Second, we are able to project maximum life expectancy for a country, say by using only information about life expectancy at the provincial level. This method could be applied in other situations where regional-only data are available but one would like to have an idea about life expectancy at a supra-regional or more aggregated level. One key theoretical challenge, however, is in dealing with the high correlation between sub regional life expectancy, and research on this problem is ongoing.

\section{Explaining the "Female Longevity Puzzle"}

\section{Malene Kallestrup-Lamb, PhD, Centre for Research in Econometric Analysis of Time-Series, Aarhus University}

This paper analyses the complexity of female longevity improvements by using detailed register data for Denmark and Norway. The aim of this paper is two-fold; first, we identify which socio-economic groups have been driving the standstill in life expectancy and analyse which causes of deaths have led 
to this standstill. Second, we investigate the forecast implications of using sub-group data and compare the performance of the single-population Lee-Carter model (Lee \& Carter, 1992) with the multi-population Li and Lee model (Li \& Lee, 2005).

It is found that the decline in life expectancy for Danish women is present for all sub-groups, however, with particular large decreases for the low-middle and middle affluence groups. We find that causes of deaths related to smoking are contributing to the slowdown in female longevity as low-middle and middle affluence groups display higher increases in death rates for smoking-related causes of deaths.

However, the following catch up effect starting from 1995 is mainly driven by the significant drop in causes of deaths relating to the circulatory system with ischaemic heart diseases. Thus, the political decision to add significant funding to this area has proven highly beneficial in terms of gains in life expectancy for females.

\section{Continuous Mortality Investigation (CMI) update on longevity modelling and high age mortality}

\section{Tim Gordon, Head of Longevity, Aon Hewitt}

\section{Steve Bale, Prudential Risk Actuary, Legal \& General, presenting on behalf of the CMI}

The Mortality Projection Committee has updated its Mortality Projection Model as follows:

- age, period and cohort components of mortality improvement are now modelled explicitly, which means they can be disaggregated into age-period and cohort components in a single step;

- users can adjust the responsiveness of the Model to new data using a single "period smoothing parameter";

- the long-term improvement rate tapers to zero earlier and quicker (between ages 85 and 110 compared to ages 90 and 120 previously); and

- although the CMI takes the view that allowing for the slope of mortality improvements ("direction of travel”) does not make for robust projections, it is now straightforward for users to incorporate this if they choose.

Since the symposium, the CMI has completed its consultation and published a revised version of the proposed model. The first official version is expected to be published in March 2017, based on England and Wales data to the end of 2016. Further details can be found in Working Papers 90, 91 and 93.

The High Age Mortality Working Party provided an update on their framework for closing mortality rate tables at very high ages. The approach focusses on graduating portfolio mortality data where there are sufficient volumes, with population mortality data being used to inform higher age behaviour where there is evidence of convergence between the two data sets. At the very highest ages, population data may not be available or credible, so expert judgement will be required to set a plausible level and shape to mortality rates. The Working Party intends to summarise its proposed approach in a working paper in 2017. 
The Working Party has also been considering the impact of modelling variants on the KannistoThatcher approach to estimating population data at high ages. These variants include modelling recent mortality trends and lowering the join age at which the Kannisto-Thatcher (Thatcher et al., 2002) approach commences. We intend to report on our findings in 2017.

\section{Stochastic mortality forecasting with smoothing and over-dispersion}

\section{Professor Jon Forster, Professor of Statistics, Department of Mathematical Sciences and ESRC Centre for Population Change, University of Southampton}

In this session, the author gave details of work carried out at Southampton University, commissioned by the Office for National Statistics, on a mortality forecasting framework. The goal of the research was to overcome several of the limitations associated with existing approaches. One such issue is those errors in models which do not adequately fit the data can lead to estimates and forecasts which over-fit fluctuations in the data and are insufficiently robust.

Another feature, lacking in many existing approaches, is the facility to impose smoothness in parameter series which vary over age, cohort and time. This is integrated into the modelling process, by the use of generalised additive models (GAMs), rather than being performed in a post hoc fashion. GAMs are a flexible class of semiparametric statistical models which allow parametric functions and unstructured (but smooth) functions of explanatory variables to appear in the model simultaneously.

The presentation explored the potential of GAMs for mortality modelling and forecasting. In particular, GAMs allow us to differentially smooth components, such as cohorts, more aggressively in areas of sparse data for the component concerned. While GAMs can provide a reasonable fit for the ages where there is adequate data, estimation and extrapolation of mortality rates using a GAM at higher ages is problematic due to high variation in crude rates. At these ages, parametric models can give a more robust fit, enabling a borrowing of strength across age groups. The presentation described how the authors extended an approach they had developed for graduating the English life table, ELT17 (Office for National Statistics), to provide a forecasting methodology based on a smooth transition between a GAM at lower ages and a fully parametric model at higher ages.

\section{On Bayesian two-population mortality models for the assessment of basis risk in longevity hedges}

\section{Andrés M. Villegas, ARC Centre of Excellence in Population Ageing Research (CEPAR), University of New South Wales, Sydney, Australia}

Index-based hedges have the potential for providing pension funds and annuity providers with an effective solution for the management of their longevity risk. However, the lack of an appropriate 
and applicable approach for quantifying the basis risk emerging from the potential mismatch between the mortality in the index reference portfolio and the pension fund/annuity book being hedged is one of the main obstacles that prevent many pension schemes and insurers from progressing in their consideration of index-based solutions. Two-population stochastic mortality models allow the joint modelling and projection of the mortality of the reference and target populations providing a possible approach to overcome this obstacle. However, often the portfolio experience data will be sparse, posing a challenge for the accurate calibration and projection of two-population models using standard estimation techniques. This presentation aimed to illustrate how a Bayesian estimation framework can be used to deal with some of the data limitation arising in the application of two-population stochastic mortality model for the assessment of basis risk in longevity hedges.

The speaker first formulated under a Bayesian framework several two-population extensions of the well-known Lee-Carter (Lee \& Carter, 1992) and Cairns-Blake-Dowd (Cairns et al., 2006) models and then contrasted the performance of the Bayesian estimation approach with that of the more traditional maximum likelihood estimation approach. This was done using illustrative hedge-effectiveness evaluation exercises while paying particular attention to the impact that different volumes of data may have on the assessment of basis risk. The results of this comparison showed that the sampling noise present in mortality data sets with limited exposures implies significant parameter estimation error in the calibration of two-population mortality models and, if not appropriately handled, can lead to a misestimation of longevity basis risk. A Bayesian estimation approach - which accounts naturally for parameter uncertainty - results in a more accurate assessment of the hedge effectiveness of index-based solutions for pension schemes with a small population.

\section{When is a cohort not a cohort? Spurious parameters in stochastic longevity models}

\section{Jon Palin, Barnett Waddingham}

Cohort effects (mortality improvements that vary by birth year) are seen in historical data for many countries. As a result, it is natural to reflect them by including cohort parameters in stochastic longevity models. However, the speaker showed that, for some models, cohort parameters are "spurious" artefacts of the model rather than reflecting genuine cohort effects.

To demonstrate this, a synthetic data set is considered. This is constructed so that it has key stylised features of historical mortality data (mortality rates that increase with age and decrease with time, with lower mortality improvements at the oldest ages) but has no cohort effect. Fitting the age-period-cohort (APC) model to this synthetic data set produces cohort parameters, even after allowing for identifiability constraints, despite the lack of cohort effects in the data.

The cohort parameters that arise from fitting the APC model to real data are similar to those from fitting to the synthetic data set. This shows that the parameters fitted to real data primarily reflect spurious features of the APC model rather than genuine cohort effects. Similar problems are seen for other models, including the Cairns-Blake-Dowd M6 model (Cairns et al., 2006) and the cohort 
extension of Lee-Carter (Lee \& Carter, 1992). Jon demonstrated algebraically how the spurious cohort parameters arise from the structure of the APC model, and how they are driven by the slope of mortality improvements by age.

Projecting these fitted parameters using the standard approach in the existing literature would give misleading results: the time-series typically used do not take account of the quadratic shape of the parameters. This finding explains the published result that the APC model produces materially higher life expectancies than some other models. This has previously been described as an example of model risk, but the speaker instead considers it to be a fundamental weakness of the structure of the APC model.

\section{Socio-economic differentials in multimorbidity and health expectancy using electronic health records: methodological challenges}

\section{Dr Madhavi Bajekal and Mei Sum Chan, Institute of Epidemiology and Health Care, University College London}

This research provided novel insights into how differences in the age of disease onset and the subsequent pace of progression to multiple diseases and death contribute to inequalities in life expectancy; and proposed that, with more people living for longer, insurers need to move beyond the single-disease analysis framework for underwriting.

Linked medical records of 1.3 million English patients aged 45+ in 2001 registered in GP practices in the Clinical Practice Research Datalink were used in the study. Patients were followed-up to March 2010. The methodological challenges (and solutions) presented included: assessing population representativeness of the sample; which, and how many, chronic diseases to include in the analysis (30 major chronic diseases were selected) and lastly, which modelling methods to use. A patient having two or more diseases concurrently (of the 30 diseases in-scope) was defined as being multimorbid.

Two models were selected and analysis performed based on a count of the number of diseases a patient has or progresses through (disease counts serving as an approximation of morbidity level):

- Cox proportional hazard model: this model calculated hazard ratios and survival probabilities to estimate life expectancies by baseline morbidity level and deprivation, adjusted for smoker status and stratified by gender. Higher levels of morbidity, being a smoker and being disadvantaged all made a significant independent contribution to the gap in life expectancy in the order listed.

- Multi-state model: this was used to partition total life expectancies into time spent with and without multimorbidity, for each deprivation, sex and smoker status sub-group. The onset of multimorbidity and death occurred at younger ages for the most deprived; but this is not attributable solely to socio-economic differences in smoking prevalence. For the same smoking status, the most deprived men still became multimorbid earlier and died at younger ages. 


\section{Parameter risk in time-series mortality forecasts}

\section{Torsten Kleinow, Heriot-Watt University, Edinburgh; Stephen Richards, Longevitas Ltd}

The ongoing macroeconomic environment of low interest rates makes life-insurer and pension scheme reserves particularly sensitive to changes in expectations of future mortality. The projection of mortality is therefore an essential part of valuing liabilities in life insurance portfolios and pension schemes. Since the future is inherently uncertain, an important tool for risk management and solvency purposes is a stochastic projection model. In their presentation and accompanying paper, Kleinow \& Richards (2016) demonstrate some practical aspects of using time-series models in assessing risk.

The first practical result is that ARIMA models can be better representations of mortality time-series than simple random-walk models. This is certainly the case for the example of males in the United Kingdom over the past 40 years. The second result relates to the sometimes-overlooked issue of parameter risk in time-series models; Kleinow \& Richards (2016) give formulae for decomposing overall risk in a forecast into undiversifiable trend risk (parameter uncertainty) and diversifiable volatility. Using the bootstrap approach from Pascual et al. (2004), Kleinow \& Richards (2016) find that, while certain kinds of parameter risk are negligible, others are too material to ignore. In the specific example of UK males, the best-fitting ARIMA model for a Lee-Carter time index also had the greatest parameter uncertainty. A consequence of this was that the best-fitting model led to the highest capital requirements for a 1-year, value-at-risk assessment of risk capital. Insurers operating under Solvency II and similar regimes therefore need to consider parameter risk in their mortality forecasting model.

A more detailed discussion can be found in Kleinow \& Richards (2016): parameter risk in time-series mortality forecasts, Scandinavian Actuarial Journal, Published online, http://dx.doi.org/ 10.1080/03461238.2016.1255655

\section{Forecasting socio-economic differences in the mortality of Danish males}

\section{Professor Andrew Cairns, Director of the Actuarial Research Centre, Institute and Faculty of Actuaries}

This research analysed the mortality of Danish males, subdivided into ten socio-economic groups, to infer mortality dynamics of different sub-groups that might be mimicked in other countries. Statistics Denmark provides a rich resource of accurate and very detailed information about all Danish residents. The research involved data for males age 55-94 in 1985-2012, and incorporated a new measure of affluence based on individual income and wealth.

The model was an extended CBD model with a non-parametric base table and it fitted well without a cohort term. The fitted model demonstrated good separation between deciles across all years and improved plausibility compared with other models that had used income instead of affluence. There 
was evidence of divergence between groups over time, despite Denmark's reputation for progressive social policies. The results were bio-demographically reasonable, as more affluent deciles had lower mortality, and mortality converged across deciles at higher ages. Results were simulated for various time periods and it was demonstrated that the correlation between a particular group and the total population increased over time, even when allowing for parameter uncertainty. The term structure of correlations was also demonstrated to be more plausible than those of some other models; this was true for individual deciles and amalgamated deciles.

The modelling framework allows a larger number of smaller sub-groups to be modelled than previously possible without losing the essential character of the underlying data. This data set and model can be used to investigate a variety of risk measurement and management problems, including basis risk and economic capital assessment.

\section{Evidence and implications of socio-economic differences in mortality}

\section{Jessica Mosher, Private Pensions Analyst, Organisation for Economic Co-operation and Development (OECD)}

This session presented the main findings of a paper published in the OECD Business and Finance Outlook 2016, which provided evidence of the differences in life expectancy at retirement for several OECD countries and discussed the implications that these differences may have for pension and annuity providers.

These differences in life expectancy present a challenge for pension and annuity providers to establish accurate mortality assumptions to value their liabilities. Using average measures of mortality for the population will likely not be accurate for subsets of the total population, yet often more granular data on mortality is not available. Further, external shocks such as changes to pension policy may affect the socio-economic characteristics of the population for which mortality assumptions are being developed, implying that historical averages may not be applicable going forward.

The tendency for pensioner and annuitant populations to be skewed towards higher economic groups also presents a challenge for providers to mitigate their longevity risk exposure. Evidence shows that not only do higher socio-economic groups live longer; they have often also experienced higher mortality improvements than those in lower socio-economic groups. This leads to a greater risk to pension and annuity providers of underestimating future mortality improvements and the mitigation of this risk becomes more expensive.

Nevertheless, differences in life expectancy may also present providers with an opportunity to better tailor pension products to different socio-economic groups, as has happened in the United Kingdom with the increased prevalence of enhanced annuities, which pay higher incomes to individuals with lower than average life expectancies. However, the discussion with workshop participants highlighted the tension between offering products priced and tailored very specifically to individual characteristics and risk profiles, and the policy objective of risk-sharing and solidarity, in particular with respect to longevity and pensions. 


\section{Should behavioural and psychological factors be used to assess mortality risk?}

\section{Peter Banthorpe, Global Head of Research and Data Analytics, RGA}

\section{Chris Falkous, Biometric Research Actuary, RGA}

When assessing mortality risk, insurers have historically focussed on traditional biometric risk factors. This session looked at two behavioural/psychological drivers of mortality - personality and subjective well-being - and considered the possibility of incorporating these into mortality risk assessment processes.

\section{Personality}

Personality can be thought of as the relatively stable set of traits, thoughts, feelings and behaviours that make a person unique. A reliable and valid way to measure personality is to assess individuals against the Big Five personality traits:

- Openness to new experiences

- Conscientiousness

- Agreeableness

- Extraversion

- Neuroticism

Research going back to the 1990s has shown that one of these - conscientiousness - is a strong predictor of mortality risk even when controlling for many factors already used in mortality risk assessment.

\section{Subjective well-being}

Subjective well-being (SWB) is research-language for the different aspects of "happiness". The evidence that SWB influences health and longevity is compelling. Researchers have found that having a strong sense of purpose and meaning in life can significantly reduce mortality risk even when controlling for many factors already used in mortality risk assessment.

\section{Data sources for objectively measuring personality and SWB}

Gathering and validating information on conscientiousness and SWB directly can be difficult. Is it possible to measure proxy objective behaviours and use these in insurance risk assessment? An example is TransUnion TrueRisk ${ }^{\circledR}$ Life, developed by RGA and TransUnion. TrueRisk ${ }^{\circledR}$ Life illustrates how credit data can be remodelled into a highly predictive differentiator of mortality risk, which reflects the fact that it is a good proxy for conscientiousness.

Three other potential data sources for objectively measuring personality and SWB were discussed:

- Social media

- Smartphones

- Wearables 
From an insurance risk assessment point of view, all have potential issues around the level of intrusiveness into peoples' lives.

The presentation finished with a review of considerations for acceptable data use in insurance applications including regulations, reasonable expectations, risks and rewards.

\section{Does money buy you longevity?}

\section{Steven Baxter, Head of Longevity Innovation and Research, Hymans Robertson LLP}

Higher-income pensioners carry disproportionate weight in pension scheme and insurance company valuations. This session focussed on developing understanding of baseline longevity, improvements and risk management solutions for high earners.

\section{Baseline longevity}

The speaker highlighted how baseline longevity rises with affluence. Using Club Vita's large proprietary data set of UK defined benefit (DB) pensioners (c. $25 \%$ of all UK DB pensioners) it was found that at low incomes life expectancy rises quickly with income. However, there comes a point where the life expectancy benefit of each $£ 1,000$ of income reduces. This relationship is clearest when using (pre-retirement) salary rather than pension as the income measure.

The benefit of using salary to measure affluence in avoiding "socio-economic drift" was highlighted. The historical lack of pension escalation alongside the accrual history within DB pension schemes means that a pension of $£ 5,000$ payable to someone currently aged 90 is - in affluence terms - much more than $£ 5,000$ payable to a current 65 -year old. Using pension amount to group individuals can overstate the life expectancy of younger pensioners, as the base table implicitly "drifts" to a higher socio-economic group at older ages.

\section{Longevity improvements}

Since the 2000s there has been a narrowing of the longevity gap between socio-economic groups. Office for National Statistics (ONS) data highlights how the gap between "managerial and professional" and "routine and manual" workers narrowed between the 1997-2001 and 2002-2006 data. However, changes to the ONS classification of socio-economic groups have limited the ability to monitor this more recently.

Club Vita has previously identified three socio-economic groups for men (two for women) who experienced profoundly differently longevity trends between 2000 and 2010. Those living in the more deprived areas (the "hard-pressed") have been catching up with the more affluent socio-economic groups (our "comfortable" group). The speaker revealed how this convergence has continued into the 2010s. Between 2000 and 2013 the gap in life expectancy at age 65 has narrowed by 0.8 years for men ( 0.4 years for women). The slower longevity improvements for the comfortable group are particularly important for pension schemes and Bulk Purchase Annuity life insurers, where typically $50 \%-80 \%$ of annual pension outgo is concentrated in the "comfortable" group. 


\section{Top-slicing risk management}

"Top-slicing", whereby the individuals representing the top (say) $20 \%$ of liabilities in a pension scheme are bought-out is a popular approach to longevity risk management. However, concerns are raised around the attractiveness of the residual liabilities for future buy-out or longevity reinsurance.

The session illustrated how, for well-designed top-slicing, there need be no anti-selection concerns.

\section{References and further reading}

Cairns, A.J.G, Blake, D. \& Dowd, K. (2006). A two-factor model for stochastic mortality with parameter uncertainty: theory and calibration. Journal of Risk and Insurance, 73(4), 687-718.

Continuous Mortality Investigation (2016). CMI Mortality Projections Model consultation. CMI Working Paper No. 90.

Continuous Mortality Investigation (2016). CMI Mortality Projections Model consultation technical paper. CMI Working Paper No. 91.

Continuous Mortality Investigation (2016). CMI Mortality Projections Model: consultation responses and plans for CMI_2016. CMI Working Paper No. 93.

Drucker, P. (1999). Management Challenges for the 21st Century. New York: Harper Collins Business.

Gillespie, D.O.S., Trotter, M.V. \& Tuljapurkar, S.D. (2016 [2014]). Divergence in age patterns of mortality change drives international divergence in lifespan inequality. Demography, 51, 1003-1017.

Gitsels, L.A., Kulinskaya, E. \& Steel, N. (2016). Survival benefits of statins for primary prevention: a cohort study. PLOS ONE, 11(11), e0166847.

Government Office for Science (2016). Foresight: Future of an ageing population, available at: https://www.gov.uk/government/collections/future-of-ageing (accessed 10 September 2017).

Haberman, S. (2016). Presentations at Salerno Longevity Symposium and Credit Suisse 7th European Pensions Conference.

Janssen, F., van Wissen, L.J.G. \& Kunst, A.E. (2013). Including the smoking epidemic in internationally coherent mortality projections. Demography, 50(4), 1341-1362.

Janssen, F. \& de Beer, J. (2016). Projecting future mortality in the Netherlands taking into account mortality delay and smoking. Joint Eurostat/UNECE Work Session on Demographic Projections, Geneva. Working Paper No. 18.

Kleinow, T. \& Richards, S.J. (2016). Parameter risk in time-series mortality forecasts, Scandinavian Actuarial Journal.

Lee, R.D. \& Carter, L.R. (1992). Modeling and forecasting US mortality. Journal of the American Statistical Association, 87(419), 659-671.

Li, N. \& Lee, R. (2005). Coherent mortality forecasts for a group of populations: an extension of the Lee-Carter method. Demography, 42(3), 575-594.

Mosher, J. (2016). Fragmentation of retirement markets due to differences in life expectancy, in OECD Business and Finance Outlook 2016. Paris, Organisation for Economic Co-Operation and Development, 177-205.

Office for National Statistics (2015). English Life Tables No. 17: 2010-2012, available at https:// www.ons.gov.uk/peoplepopulationandcommunity/birthsdeathsandmarriages/lifeexpectancies/ bulletins/englishlifetablesno17/2015-09-01 (accessed 10 September 2017).

Office for National Statistics: Health and Life Expectancies, available at https://www.ons.gov.uk/ peoplepopulationandcommunity/healthandsocialcare/healthandlifeexpectancies (accessed 10 September 2017). 
Office for National Statistics (2016). Standard Occupational Classification (SOC 2000) and NS-SEC on the labour force survey, available at https://www.ons.gov.uk/employmentand labourmarket/peopleinwork/employmentandemployeetypes/methodologies/standardoccupation alclassificationsoc2000andnsseconthelabourforcesurvey (accessed 10 September 2017).

Olshansky, S.J., Martin, G.M. \& Kirkland, J.L. (ed) (2016). Aging: The Longevity Dividend. Cold Spring Harbor Perspectives in Medicine, Cold Spring Harbor, NY: Cold Spring Harbor Laboratory Press.

Pascual, L., Romo, J. \& Ruiz, E. (2004). Bootstrap predictive inference for ARIMA processes. Journal of Time Series Analysis, 25(4), 449-465.

Thatcher, A.R., Kannisto, V. \& Andreev, K.F. (2002). The survivor ratio method for estimating numbers at high ages. Demographic Research, 6, 1-18.

TrueRisk $^{\circledR}$ Life. TransUnion TrueRisk ${ }^{\circledR}$ Life, developed by RGA and TransUnion.

White, K.M. (2002). Longevity advances in high-income countries, 1955-96. Population and Development Review, 28(1), 59-76. 